\title{
FINITE TIME EXTINCTION FOR SOLUTIONS TO FAST DIFFUSION STOCHASTIC POROUS MEDIA EQUATIONS
}

\author{
VIOREL BARBU, GIUSEPPE DA PRATO, AND MICHAEL RÖCKNER
}

ABSTRACT. We prove that the solutions to fast diffusion stochastic porous media equations have finite time extinction with strictly positive probability.

\section{INTRODUCTION}

Consider the stochastic porous media equation (1) $\left\{\begin{array}{l}d X(t)-\rho \Delta\left(|X|^{\alpha}(t) \operatorname{sign} X(t)\right) d t-\Delta(\tilde{\Psi}(X(t)) d t=\sigma(X(t)) d W(t), \text { in }(0, \infty) \times \mathcal{O}, \\ X=0 \quad \text { on }(0, \infty) \times \partial \mathcal{O}, \quad X(0, x)=x \quad \text { on } \mathcal{O},\end{array}\right.$

where $\rho>0, \alpha \in(0,1), \tilde{\Psi}$ is a continuous monotonically non decreasing function of linear growth and $\sigma(X) d W=\sum_{k=1}^{\infty} \mu_{k} X e_{k} d \beta_{k}, \quad t \geq 0$, where $\left\{\beta_{k}\right\}$ is a sequence of independent real Brownian motions on a filtered probability space $\left(\Omega, \mathcal{F},\left\{\mathcal{F}_{t}\right\}, \mathbb{P}\right)$ and $\left\{e_{k}\right\}$ is an orthormal basis in $L^{2}(\mathcal{O})$ which for convenience will be taken as the eigenfunction system for the Laplace operator with Dirichlet boundary conditions, i.e., $-\Delta e_{k}=\lambda_{k} e_{k}$ in $\mathcal{O}, e_{k}=0$ on $\partial \mathcal{O}$, where $\mathcal{O}$ is an open and bounded subset of $\mathbb{R}^{d}$, with smooth boundary $\partial \mathcal{O}$. We shall assume that $\sum_{k=1}^{\infty} \mu_{k}^{2} \lambda_{k}^{2}<\infty$. Equation (11) for $0<\alpha<1$ is relevant in the mathematical modelling of the dynamics of an ideal gas in a porous medium and, in particular, in a plasma fast diffusion model (for $\alpha=1 / 2$ ) (see e.g. [4]). The existence and uniqueness of a strong solution in the sense to be defined below was studied in [1, [2, $[3,[5]$ for more general nonlinear stochastic equations of the form (11). In [3] (see also [1]) it was also proven that for $\alpha=0$ and $d=1$ the solution $X=X(t, x)$ to (11) has the finite extinction property: $\mathbb{P}(\tau \leq n) \geq 1-\frac{|x|_{-1}}{\rho \gamma}\left(\int_{0}^{n} e^{-C_{N} s} d s\right)^{-1}$ for $|x|_{-1}<C_{N}^{-1} \rho \gamma$ where $\tau=\inf \left\{t \geq 0:|X(t, x)|_{-1}=0\right\}=\sup \left\{t \geq 0:|X(t, x)|_{-1}>0\right\}$ and $C_{N}, \gamma$ are constants related to the Wiener process $W$ and respectively to the domain $\mathcal{O} \subset \mathbb{R}^{1}$.

The following notations will be used in the sequel. $H=L^{2}(\mathcal{O}), p \geq 1$, with the norm denoted by $|\cdot|_{2}$ and scalar product $\langle\cdot, \cdot\rangle \cdot H^{-1}(\mathcal{O})$ is the dual of the Sobolev space $H_{0}^{1}(\mathcal{O})$ and is endowed with the scalar product $\langle u, v\rangle_{-1}=\left\langle u,(-\Delta)^{-1} v\right\rangle$, where $\Delta$ is the Laplace operator with domain $H^{2}(\mathcal{O}) \cap H_{0}^{1}(\mathcal{O})$. All processes $X=$ $X(t)$ arising here are adapted with respect to the filtration $\left\{\mathcal{F}_{t}\right\}$. For a Banach space $E, L_{W}^{p}(0, T ; E)$ denotes the space of all adapted processes in $L^{p}(0, T ; E)$. We shall use standard notation for Sobolev spaces and spaces of integrable functions on $\mathcal{O}$. 


\section{The MAIN RESUlT}

Definition 2.1. Let $x \in H$. An $H$-valued continuous $\left(\mathcal{F}_{t}\right)$-adapted process $X=$ $X(t, x)$ is called a solution to (1) on $[0, T]$ if $X \in L^{p}(\Omega \times(0, T) \times \mathcal{O}) \cap L^{2}\left(0, T ; L^{2}(\Omega, H)\right), p \geq$ 2 , such that $\mathbb{P}$-a.s. $\forall j \in \mathbb{N}, t \in[0, T]$,

$$
\begin{aligned}
\left\langle X(t, x), e_{j}\right\rangle= & \left\langle x, e_{j}\right\rangle+\int_{0}^{t} \int_{\mathcal{Q}}\left(\rho|X(s, x)(\xi)|^{\alpha} \operatorname{sign} X(s, x)(\xi)+\tilde{\Psi}(X(s, x)(\xi))\right) \Delta e_{j}(\xi) d \xi d s \\
& +\sum_{k=1}^{\infty} \mu_{k} \int_{0}^{t}\left\langle X(s, x) e_{k}, e_{j}\right\rangle d \beta_{k}(s),
\end{aligned}
$$

For $x \in L^{p}(\mathcal{O}), p \geq 4$ and $d=1,2,3$ there is a unique solution $X \in L_{W}^{\infty}\left(0, T ; L^{p}(\Omega, H)\right)$ to (11) in the sense of Definition 2.1. Moreover, if $x \geq 0$ a.e. in $\mathcal{O}$ then $X \geq 0$ a.e. in $\Omega \times[0, T] \times \mathcal{O})$.

By the proof of [3, Theorem 2.2] and [3, Proposition 3.4] we also know that for $\lambda \rightarrow 0$,

$$
\left\{\begin{array}{l}
X_{\lambda} \rightarrow X \text { strongly both in } L^{2}\left(0, T ; L^{2}\left(\Omega, L^{2}(\mathcal{O})\right)\right) \text { and in } L^{2}(\Omega ; C([0, T] ; H)), \\
\text { weakly in } L^{p}(\Omega \times(0, T) \times \mathcal{O}), \text { and } \text { weak }^{*} \text { in } L^{\infty}\left(0, T ; L^{p}\left(\Omega ; L^{p}(\mathcal{O})\right)\right)
\end{array}\right.
$$

where $X_{\lambda}, \lambda>0$, is the solution to approximating equation

(4)

$$
\left\{\begin{array}{l}
d X_{\lambda}(t)-\Delta\left(\Psi_{\lambda}\left(X_{\lambda}(t)\right)+\lambda X_{\lambda}(t)+\tilde{\Psi}\left(X_{\lambda}(t)\right)\right) d t=\sigma\left(X_{\lambda}(t)\right) d W(t) \\
\Psi_{\lambda}\left(X_{\lambda}\right)+\lambda X_{\lambda}+\tilde{\Psi}\left(X_{\lambda}\right)=0 \quad \text { on } \partial \mathcal{O}, \quad X_{\lambda}(0, x)=x \\
\Psi_{\lambda}(x)=\frac{1}{\lambda}\left(x-\left(1+\lambda \Psi_{0}\right)^{-1}(x)\right)=\Psi_{0}\left(\left(1+\lambda \Psi_{0}\right)^{-1}(x)\right), \quad \Psi_{0}(x)=\rho|x|^{\alpha} \operatorname{sign} x
\end{array}\right.
$$

Everywhere in the sequel $X=X(t, x)$ is the solution to (11) in the sense of Definition 2.1 where $x \in L^{4}(\mathcal{O})$. Below $\gamma$ shall denote the minimal constant arising in the Sobolev embedding $L^{\alpha+1}(\mathcal{O}) \subset H^{-1}(\mathcal{O})$ (see (7) below) and $C^{*}=$ $\sum_{k=1}^{\infty} \mu_{k}^{2}\left|e_{k}\right|_{H_{0}^{1}(\mathcal{O})}^{2}=\sum_{k=1}^{\infty} \mu_{k}^{2} \lambda_{k}^{2}$. Theorem 2.2 is the main result of the paper.

Theorem 2.2. Assume that $d=1,2,3$ and that $0<\alpha<1$ if $d=1,2, \frac{1}{5} \leq \alpha<$ 1 if $d=3$. Let $\tau:=\inf \left\{t \geq 0:|X(t, x)|_{-1}=0\right\}$. Then we have $|X(t, x)|_{-1}=$ 0 , for $t \geq \tau$, $\mathbb{P}$-a.s.. Furthermore $\mathbb{P}(\tau \leq t) \geq 1-\frac{|x|_{-1}^{1-\alpha}}{(1-\alpha) \rho \gamma^{1+\alpha}}\left(\int_{0}^{t} e^{-(1-\alpha) C^{*} s} d s\right)^{-1}$. In particular, if $|x|_{-1}^{1-\alpha}<\frac{\rho \gamma^{1+\alpha}}{C^{*}}$, then $\mathbb{P}(\tau<\infty)>0$, and if $C^{*}=0$, then $\tau \leq$ $\frac{|x|_{-1}^{1-\alpha}}{(1-\alpha) \rho \gamma^{1+\alpha}}$.

Remark 1. This result extends to $\mathcal{O} \subset \mathbb{R}^{d}$ with $d \geq 4$, if $\alpha \in\left[\frac{d-2}{d+2}, 1\right)$. However, we have to strengthen the assumption on $\mu_{k}, k \in \mathbb{N}$, see [1, Section 4] and in particular [6, Remark 2.9(iii)] for a detailed discussion.

\section{Proof of Theorem 2.2}

We shall proceed as in the proof of [3, Theorem 4.2]. Consider the solution $X_{\lambda} \in L_{W}^{2}\left(0, T ; L^{2}\left(\Omega ; H_{0}^{1}(\mathcal{O})\right)\right)$ to equation (4). Then by applying the classical Itô formula to the real valued semi-martingale $\left|X_{\lambda}(t)\right|_{-1}^{2}, t \in[0, T]$, and to the function 
FINITE TIME EXTINCTION FOR SOLUTIONS TO FAST DIFFUSION STOCHASTIC POROUS MEDIA EQUATIONS $\varphi_{\varepsilon}(r)=\left(r+\varepsilon^{2}\right)^{(1-\alpha) / 2}, \quad r \in \mathbb{R}$, we find that

$(5)$

$$
\begin{aligned}
& d \varphi_{\varepsilon}\left(\left|X_{\lambda}(t)\right|_{-1}^{2}\right)+(1-\alpha)\left(\left|X_{\lambda}(t)\right|_{-1}^{2}+\varepsilon^{2}\right)^{-(1+\alpha) / 2}\left\langle X_{\lambda}(t), \Psi_{\lambda}\left(X_{\lambda}(t)\right)+\lambda X_{\lambda}(t)+\tilde{\Psi}_{\lambda}\left(X_{\lambda}(t)\right)\right\rangle d t \\
& =\frac{1}{2} \sum_{k=1}^{\infty} \mu_{k}^{2}(1-\alpha) \frac{\left.\left|X_{\lambda}(t) e_{k}\right|_{-1}^{2}\left(\left|X_{\lambda}(t)\right|_{-1}^{2}+\varepsilon^{2}\right)-(1-\alpha)^{2}\left|\left\langle X_{\lambda}(t) e_{k}, X_{\lambda}(t)\right\rangle_{-1}\right|^{2}\right)}{\left(\left|X_{\lambda}(t)\right|_{-1}^{2}+\varepsilon^{2}\right)^{(3+\alpha) / 2}} d t \\
& +\left\langle\sigma\left(X_{\lambda}(t)\right) d W(t), \varphi_{\varepsilon}^{\prime}\left(\left|X_{\lambda}(t)\right|_{-1}^{2}\right) X_{\lambda}(t)\right\rangle_{-1} \\
& \leq \frac{1}{2} \sum_{k=1}^{\infty} \mu_{k}^{2} \frac{(1-\alpha)\left|X_{\lambda}(t) e_{k}\right|_{-1}^{2}}{\left(\left|X_{\lambda}(t)\right|_{-1}^{2}+\varepsilon^{2}\right)^{(1+\alpha) / 2}} d t+\left\langle\sigma\left(X_{\lambda}(t)\right) d W(t), \varphi_{\varepsilon}^{\prime}\left(\left|X_{\lambda}(t)\right|_{-1}^{2}\right) X_{\lambda}(t)\right\rangle_{-1} \\
& \leq C^{*} \frac{(1-\alpha)\left|X_{\lambda}(t) e_{k}\right|_{-1}^{2}}{\left(\left|X_{\lambda}(t)\right|_{-1}^{2}+\varepsilon^{2}\right)^{(1+\alpha) / 2}} d t+\left\langle\sigma\left(X_{\lambda}(t)\right) d W(t), \varphi_{\varepsilon}^{\prime}\left(\left|X_{\lambda}(t)\right|_{-1}^{2}\right) X_{\lambda}(t)\right\rangle_{-1}
\end{aligned}
$$

Then letting $\lambda \rightarrow 0$, by (3) we get that $\liminf _{\lambda \rightarrow 0} \int_{0}^{T}\left\langle\Psi_{\lambda}\left(X_{\lambda}(t)\right), X_{\lambda}(t)\right\rangle d t \geq \rho \int_{0}^{T}|X(t)|_{L^{1+\alpha}(\mathcal{O})}^{1+\alpha} d t$, $\mathbb{P}$-a.s. and hence

(6)

$$
\begin{aligned}
& \varphi_{\varepsilon}\left(|X(t)|_{-1}^{2}\right)+(1-\alpha) \rho \int_{r}^{t} \frac{|X(s)|_{L^{\alpha+1}(\mathcal{O})}^{\alpha+1}}{\left(|X(s)|_{-1}^{2}+\varepsilon^{2}\right)^{(1+\alpha) / 2}} d s \leq \varphi_{\varepsilon}\left(|X(r)|_{-1}^{2}\right) \\
& +C^{*} \int_{r}^{t} \frac{(1-\alpha)|X(s)|_{-1}^{2}}{\left(|X(s)|_{-1}^{2}+\varepsilon^{2}\right)^{(1+\alpha) / 2}} d s+2 \int_{r}^{t}\left\langle\sigma(X(s)) d W(s), \varphi_{\varepsilon}^{\prime}\left(|X(s)|_{-1}^{2}\right) X(s)\right\rangle_{-1}, \quad \mathbb{P} \text {-a.s., } r<t .
\end{aligned}
$$

Next by the Sobolev embedding theorem we have (7)

$$
|u|_{-1} \leq \gamma|u|_{L^{\alpha+1}(\mathcal{O})}, \forall u \in L^{\alpha+1}(\mathcal{O}) \text {, if } d>2 \text { and } \alpha \geq \frac{d-2}{d+2} \text {, and } \forall \alpha>0 \text {, if } \mathrm{d}=1,2 \text {. }
$$

Then substituting (7) into (6) we get

$$
\begin{aligned}
& \varphi_{\varepsilon}\left(|X(t)|_{-1}^{2}\right)+(1-\alpha) \rho \gamma^{1+\alpha} \int_{r}^{t} \frac{|X(s)|_{-1}^{\alpha+1}}{\left(|X(s)|_{-1}^{2}+\varepsilon^{2}\right)^{(1+\alpha) / 2}} d s \leq \varphi_{\varepsilon}\left(|X(r)|_{-1}^{2}\right) \\
& +C^{*} \int_{r}^{t} \frac{(1-\alpha)|X(s)|_{-1}^{2}}{\left(|X(s)|_{-1}^{2}+\varepsilon^{2}\right)^{(1+\alpha) / 2}} d s+\int_{r}^{t}\left\langle\sigma(X(s)) d W(s), \varphi_{\varepsilon}^{\prime}\left(|X(s)|_{-1}^{2}\right) X(s)\right\rangle_{-1}, \quad \mathbb{P} \text {-a.s., } r<t .
\end{aligned}
$$

Now for $\epsilon \rightarrow 0$ we have

$$
\begin{aligned}
& |X(t)|_{-1}^{1-\alpha}+(1-\alpha) \rho \gamma^{1+\alpha} \int_{r}^{t} 1_{\left\{|X(s)|_{-1}>0\right\}} d s \leq|X(r)|_{-1}^{1-\alpha}+C^{*}(1-\alpha) \int_{r}^{t}|X(s)|_{-1}^{1-\alpha} d s \\
& +(1-\alpha) \int_{r}^{t}\left\langle\sigma(X(s)) d W(s),|X(s)|_{-1}^{-(\alpha+1)} X(s)\right\rangle_{-1}, \mathbb{P} \text {-a.s., } r<t .
\end{aligned}
$$

Hence by Itô's product rule

$$
\begin{aligned}
& e^{-C^{*}(1-\alpha) t}|X(t)|_{-1}^{1-\alpha}+(1-\alpha) \rho \gamma^{1+\alpha} \int_{r}^{t} e^{-C^{*}(1-\alpha) s} 1_{\left\{|X(s)|_{-1}>0\right\}} d s \leq e^{-C^{*}(1-\alpha) r}|X(r)|_{-1}^{1-\alpha} \\
& +(1-\alpha) \int_{r}^{t} e^{-C^{*}(1-\alpha) s} 1_{\left\{|X(s)|_{-1}>0\right\}}\left\langle\sigma(X(s)) d W(s),|X(s)|_{-1}^{-(\alpha+1)} X(s)\right\rangle_{-1}, \quad \mathbb{P} \text {-a.s., } r<t .
\end{aligned}
$$

From this it immediately follows that $e^{-C^{*}(1-\alpha) t}|X(t)|_{-1}^{1-\alpha}, \quad t \geq 0$, is an $\left(\mathcal{F}_{t}\right)$ supermartingale, hence $|X(t)|_{-1}=0$ for all $t \geq \tau$. So, (9) with $r=0$ after taking expectation implies that $\int_{0}^{t} e^{-C^{*}(1-\alpha) s} \mathbb{P}(\tau>s) d s \leq \frac{|x|_{-1}^{1-\alpha}}{(1-\alpha) \rho \gamma^{1+\alpha}}, \quad t \geq 0$. This implies that $\mathbb{P}(\tau>t) \leq \frac{|x|_{-1}^{1-\alpha}}{(1-\alpha) \rho \gamma^{1+\alpha}}\left(\int_{0}^{t} e^{-C^{*}(1-\alpha) s} d s\right)^{-1}, t \geq 0$, and the assertion follows. 


\section{ACKNOWLEDGEMENT}

This work has been supported in part by the PIN-II ID-404 (2007-2010) project of Romanian Minister of Research, the DFG -International Graduate School "Stochastics and Real World Models",the SFB-701 and the BiBoS-Research Center.', the research programme "Equazioni di Kolmogorov" from the Italian "Ministero della Ricerca Scientifica e Tecnologica" and "FCT, POCTI-219, FEDER".

\section{REFERENCES}

[1] V. Barbu, G. Da Prato and M. Röckner, Existence and uniqueness of nonnegative solutions to the stochastic porous media equation, Indiana University Math. Journal, 57, 187-212, 2008.

[2] V. Barbu, G. Da Prato and M. Röckner, Existence of strong solutions for stochastic porous media equation under general monotonicity conditions, Annals of Probability. (to appear).

[3] V. Barbu, G. Da Prato and M. Röckner, Stochastic porous media equations and self-organized criticality, Comm. Math. Phys, (to appear).

[4] J. Berryman and C. Holland, Stability of the separable solution for fast diffusion. Arch. Rational Mech. Anal. 74, no. 4, 379-388, 1980.

[5] J. Ren, M. Röckner and F.Y. Wang, Stochastic generalized porous media and fast diffusion equations. J. Differential Equations 238, no. 1, 118-152, 2007.

[6] M. Röckner and F.Y. Wang, Non-monoton Stochastic generalized porous media equations. Preprint, J. Differential Equations (to appear).

E-mail address: vb41@uaic.ro

Institute of Mathematics "Octav Mayer", Iasi, Romania

E-mail address: daprato@sns.it

Scuola Normale Superiore di Pisa, Italy

E-mail address: roeckner@Mathematik. Uni-Bielefeld.DE

Faculty of Mathematics, University of Bielefeld, Germany and Department of Mathematics and Statistics, Purdue University,, U. S. A 